\title{
Pathophysiology of Benign Prostatic Hyperplasia and Benign Prostatic Enlargement: A Mini-Review
}

\author{
Stephan Madersbacher ${ }^{a, b}$ Natalie Sampson ${ }^{c}$ Zoran Culig $^{c}$ \\ ${ }^{a}$ Department of Urology, Kaiser Franz Josef Hospital, Vienna, Austria; ${ }^{b}$ Sigmund Freud Private University, Vienna, \\ Austria; ${ }^{C}$ Department of Urology, Medical University of Innsbruck, Innsbruck, Austria
}

\section{Keywords}

Benign prostatic hyperplasia - Benign prostatic

enlargement · Pathophysiology · Androgens · Metabolic

syndrome · Management

\begin{abstract}
Benign prostatic hyperplasia (BPH), benign prostatic enlargement (BPE) and lower urinary tract symptoms (LUTS) belong to the most frequent diseases in ageing men. Beyond the 6 th decade of life, more than $30 \%$ of men suffer from moderate to severe LUTS requiring intervention. The pathophysiology of $\mathrm{BPH} / \mathrm{BPE}$ is still incompletely understood. The dominant role of the androgen system and the androgen receptor is well defined. Androgen receptors are expressed in BPH tissue in which they are activated by the potent androgen dihydrotestosterone. Synthesis of dihydrotestosterone is under control of the 5a-reductase enzyme, activity of which is antagonized by finasteride and dutasteride. More recently, the impact of prostatic inflammation and metabolic parameters particularly for the development of BPE and LUTS has increasingly been recognized. A better understanding of the pathophysiology is a prerequisite for the development of novel, more effective medical treatment options.

(c) 2019 S. Karger AG, Basel
\end{abstract}

\section{Introduction}

Benign prostatic hyperplasia $(\mathrm{BPH})$ belongs to the most frequent diseases in ageing men. In the 4th decade of life, $\mathrm{BPH}$ is demonstrable in $30-40 \%$ of men, and its prevalence increases almost linearly to $70-80 \%$ in those older than 80 years. BPH, however, is a purely histological definition and must be distinguished from benign prostatic enlargement (BPE), which describes an enlarged prostate, and lower urinary tract symptoms (LUTS), which usually lead to medical consultation [1]. LUTS can be separated into storage symptoms (urgency, frequency, nocturia and urge urinary incontinence), voiding symptoms (reduced flow and feeling of incomplete emptying) and post-void dribbling. LUTS are best quantified by validated questionnaires, such as the International Prostate Symptom Score (IPSS) or the American Urological Association (AUA) symptom score [1]. LUTS in elderly men are dominantly caused by urodynamic changes of the lower urinary tract, such as benign prostatic obstruction and detrusor overactivity or underactivity [2].

In parallel to $\mathrm{BPH}$, also BPE, LUTS and abnormal urodynamic patterns increase with age. The prevalence of moderate/severe LUTS (IPSS $>7$ ) is around $20 \%$ in the

\section{KARGER}

(c) 2019 S. Karger AG, Basel

E-Mail karger@karger.com

www.karger.com/ger
Stephan Madersbacher, MD, FEBU

Department of Urology, Kaiser Franz Josef Hospital

Kundratstrasse 3

AT-1100 Vienna (Austria)

E-Mail stephan.madersbacher@wienkav.at 
5 th, $30 \%$ in the 6 th and $40 \%$ in the 8 th decade of life. In a prevalence study based on 2,096 men living in Austria, it was calculated that currently approximately 350,000 men older than 40 years living in Austria (total population 8.7 million) have moderate to severe LUTS [3]. Due to demographic changes, this figure will rise substantially to approximately 500,000 in the next 2 decades, emphasising also its socioeconomic relevance [3].

Despite its high prevalence and socioeconomic impact, the pathophysiology of $\mathrm{BPH}$ is only incompletely understood. It is, for example, still largely unknown why some men develop a $40 \mathrm{-g}$ prostate and others a $200 \mathrm{-g}$ prostate. Herein, we review several pathophysiological (with emphasis on the androgen pathway) and the most relevant clinical aspects of BPH and BPE. The pathomechanisms leading to LUTS are much more complex than just BPH/BPE and involve several urodynamic patterns (e.g., detrusor overactivity/underactivity), changes within the urothelium and bladder ultrastructure, receptor status of the anticholinergic system, pelvic ischaemia and many more [4]. This extensive field is not covered in this review, and the interested reader is referred to the respective literature [4].

\section{Androgen Pathway}

The prostate, like other sex-accessory tissues, is stimulated in its growth, maintenance and secretory function by the continued presence of certain hormones and growth factors. Foremost among these is testosterone. Serum testosterone is under the control of the hypothalamic (LHRH)pituitary (LH/FSH)-testicular (testosterone) hormone axis. Testosterone originating from the testis $(95 \%)$ and adrenal gland (5\%) is the major serum androgen stimulating prostate growth. The average testosterone concentration in the plasma is approximately $600 \mathrm{ng} / \mathrm{mL}$. Serum testosterone levels remain fairly constant between 25 and 60 years of age, yet decline gradually thereafter. Although testosterone is the primary plasma androgen, it appears to function as a prohormone in that the most active form of the androgen in the prostate is dihydrotestosterone (DHT) (Fig. 1). Hormonal regulation of $\mathrm{BPH}$ is dependent on the presence of androgen and oestrogen receptors. In addition, activity of the enzyme $5 a$-reductase plays an important role in the $\mathrm{BPH}$ pathogenesis.

Androgen receptors (AR) are widely expressed in benign epithelium and adjacent stroma. The action of AR in prostate tissue is enhanced by coactivators which may interact with $\mathrm{N}$-terminal, DNA-binding and/or ligand- binding domains of the receptor. Importantly, alterations in $\mathrm{AR}$ expression comparable to those observed in cancer tissue have not been observed in benign disorders. Thus, there are no reports in the literature on AR gene amplifications, mutations or enhanced interaction with coactivators in BPH. Differences in the number of polymorphic CAG repeats in the $\mathrm{N}$-terminal region of the AR structure have been reported in the literature; however, there is no consensus in the scientific community on the clinical relevance of these polymorphisms in the pathogenesis of $\mathrm{BPH} / \mathrm{LUTS}$. It is established that receptors with a lower number of CAG repeats have a higher transcriptional activity. Shorter CAG repeats in BPH patients in Finland were less common, contrary to expectations [5]. It seems that this issue is more relevant to understanding racial differences in prostate cancer rather than $\mathrm{BPH}$.

In order to understand the basis for therapeutic interference in $\mathrm{BPH}$, it is important to emphasize that $5 a$-reductase is responsible for the synthesis of a more potent androgen, DHT. Therefore, inhibitory approaches in $\mathrm{BPH}$ primarily target $5 \mathrm{a}$-reductase. $\mathrm{BPH}$ tissue contains oestrogen receptor $\alpha$ in stroma and oestrogen receptor $\beta$ in epithelium. Epigenetic alterations that lead to changes in oestrogen receptor $\beta$ expression have been described in primary and metastatic cancer tissue. Thus, one could conclude that there are no alterations in the expression of steroid receptors that considerably affect benign hyperplastic tissue. However, since the more potent androgen DHT is generated in hyperplastic tissue, the use of anti-hormonal agents, which suppress functional activity of the AR, on the basis of pre-clinical findings will be further discussed.

In this context, it is interesting to mention that the neuropeptide serotonin (5-HT) down-regulates AR, thus preventing prostate branching [6]. The experiments with different 5-HT agonists yielded similar results regarding the androgen signalling pathway. 5-HT, which is a constituent of neuroendocrine cells, is down-regulated in benign prostate tissue. It is a general opinion that neuroendocrine cells contribute to the progression of prostate cancer, whereas in $\mathrm{BPH}$, they could have a different effect. Another compound which attenuates the effects of testosterone on BPH tissue is vanillic acid [7]. It was demonstrated that treatment with vanillic acid, which is also known as an anti-inflammatory agent, leads to reduction of prostate weight and induction of epithelial thickness. Vanillic acids was shown to inhibit $A R$, oestrogen receptor $\alpha$ and steroid receptor coactivator 1. The authors have also performed in vitro experiments which largely confirmed the in vivo data. $\mathrm{BPH}$ medical treatment with the $5 a$-reductase type II inhibitor finasteride 
Fig. 1. Hormonal regulation of $\mathrm{BPH}$. The potent androgen DHT is synthesized from testosterone, and the process is under control of $5 a$-reductase. DHT regulates expression of growth-promoting and prodifferentiation genes in benign prostate cancer, in part in coordination with the IGF pathway. The effects of DHT could be antagonized by finasteride and dutasteride and the effects of IGF by metformin. AR, androgen receptor; $\mathrm{BPH}$, benign prostatic hyperplasia; DHT, dihydrotestosterone; IGF, insulin-like growth factor.

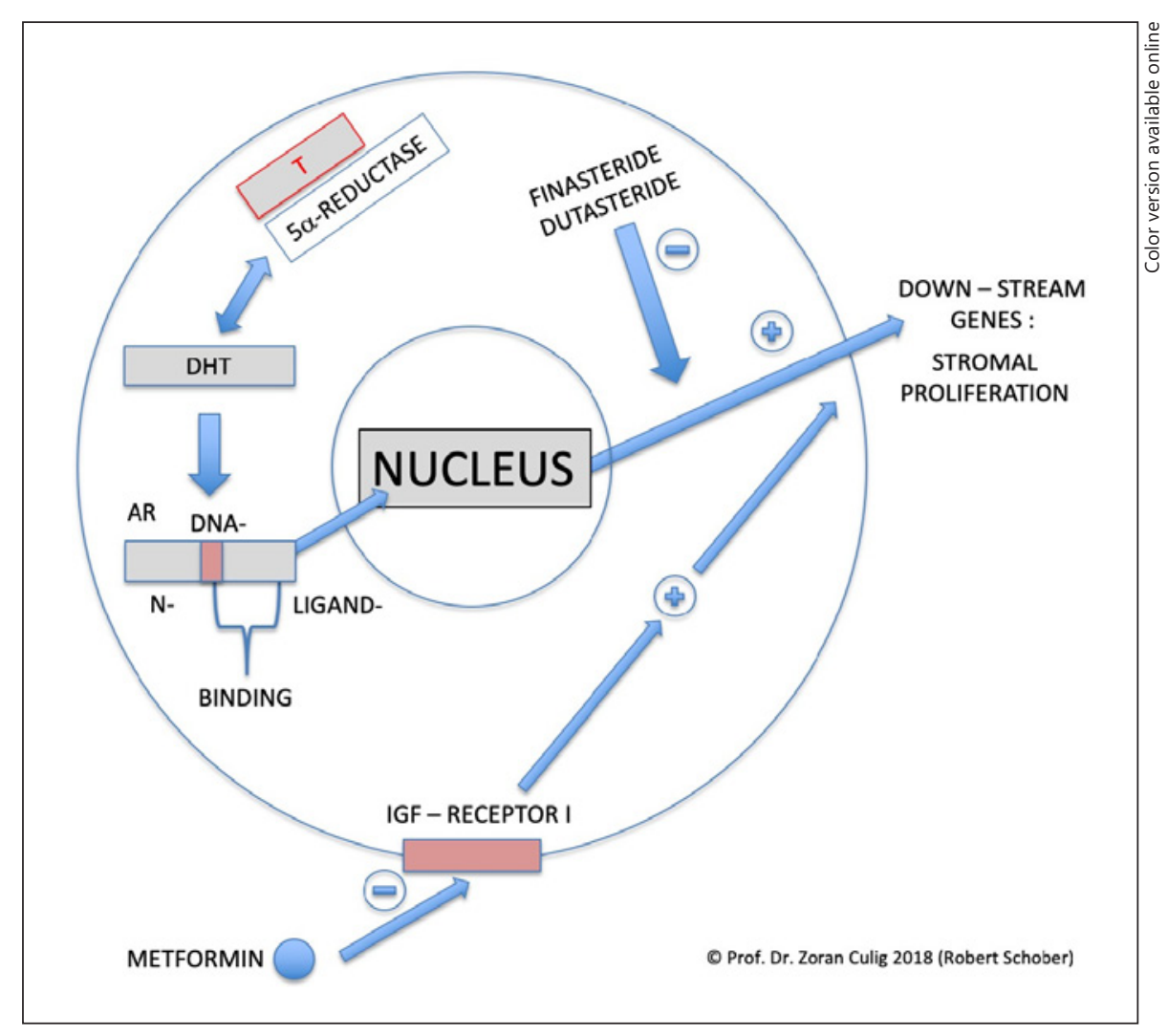

is a standard clinical procedure so that there is no need to discuss the experimental basis and early studies in this field in detail. However, it should be mentioned that investigations on the oncogenes Bcl-2 and Bcl-xl in BPH tissue after finasteride treatment have been carried out [8]. No effect of finasteride was reported, thus suggesting that $5 \alpha$-reductase inhibition does not parallel inhibition of these 2 members of the Bcl family. Interestingly, it has been proposed that the presence of the oncogene c-Jun in prostate fibroblasts is required for the in vitro growth of BPH-1 cells. Finasteride inhibitory effects on cellular proliferation were observed in the presence of c-Jun [9]. Consistent with previous preclinical studies with finasteride, a therapeutic basis for the use of the $5 \alpha$-reductase inhibitor dutasteride in benign and malignant prostate disorders has been established [10]. Concomitant use of dutasteride and testosterone led to growth inhibition of BPH-1 cells.

There are several options to interfere with androgen-induced growth of $\mathrm{BPH}$ cells when different nutrition ingredients or compounds that regulate cellular metabolism are used. For example, the widely used anti-diabetic agent metformin, which is known for its anti-proliferative and proapoptotic properties, was demonstrated to attenuate testos- terone-induced $\mathrm{BPH}$ in rats [11]. Metformin antagonized the effects of testosterone on the up-regulation of oestrogen receptor $\alpha$ and the down-regulation of oestrogen receptor $\beta$ in an animal model. Although metformin did not cause any effect on $5 a$-reductase, it decreases mRNA for insulinlike growth factor (IGF)-I and its receptor. Consequently, metformin acted as a pro-apoptotic factor through inhibition of the Akt pathway. IGFs regulate cell proliferation and apoptosis through a complex system which depends on the expression of receptors and binding proteins. Expression of both IGFs is increased in human BPH tissue [12]. Interestingly, higher levels of IGF binding protein-3 were measured in the stromal compartment of the prostate [13]. Importantly, this binding protein potentiated stromal remodelling by transforming growth factor (TGF) $-\beta$ by a mechanism that is independent of IGF. On the basis of these findings, it could be concluded that the IGF axis is a valid target for novel therapy approaches for BPH.

Hormonal regulation of $\mathrm{BPH}$ could be antagonized by chemopreventive agents. The term "chemopreventive" is in most cases used in connection with cancer prevention; however, the in vitro and in vivo effects of these compounds on benign tissue are of considerable interest. In 
Fig. 2. The potential role of the metabolic syndrome in the pathophysiology of BPE/ LUTS. BPE, benign prostatic enlargement; $\mathrm{BPH}$, benign prostatic hyperplasia; LUTS, lower urinary tract symptoms.

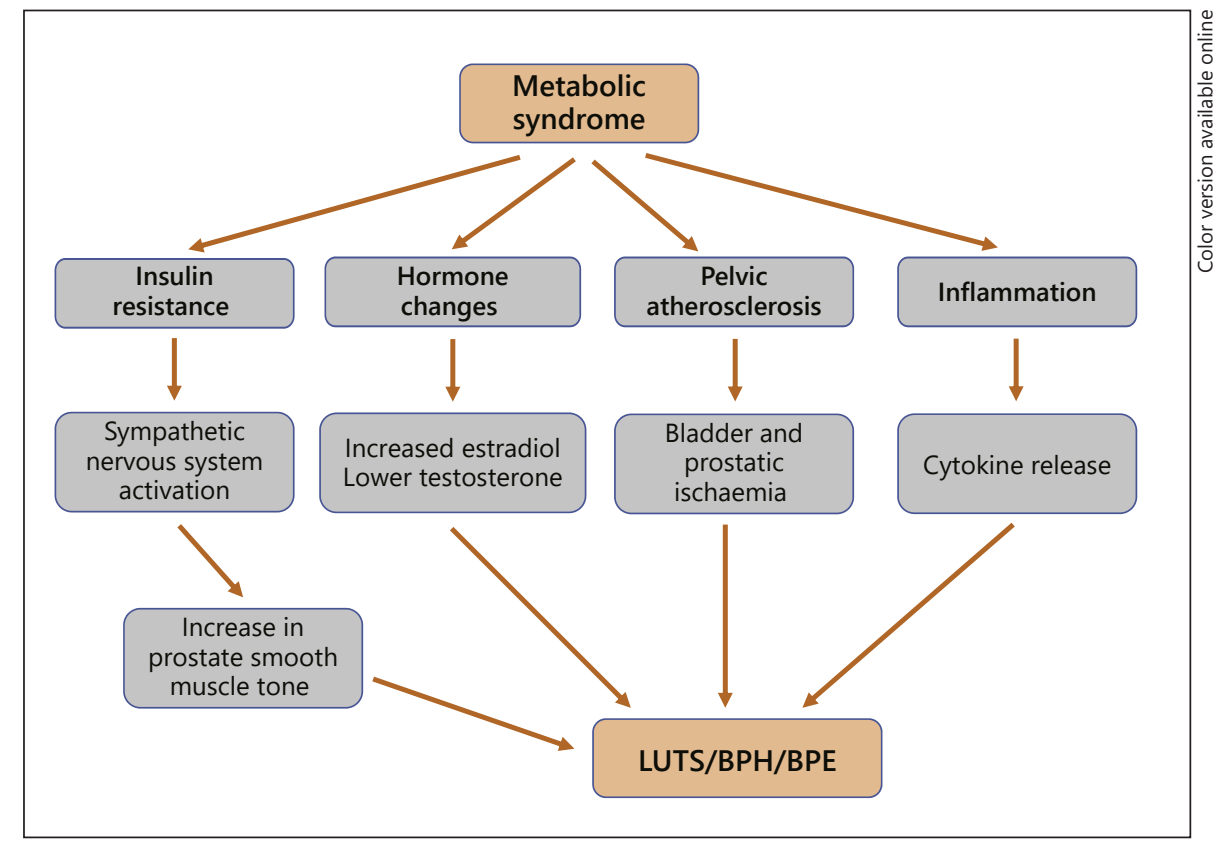

case of $\mathrm{BPH}$, it would be more appropriate to use the term "dietary compounds." They include polyphenols, flavonoids and isoflavonoids. They are contained in fruits, broccoli, and green tea. Pomegranate fruit extract, which has anti-inflammatory and anti-oxidative properties, reduced testosterone increase in prostate weight and prevented hormonally induced histological changes [14]. Similarly, ginseng, which is used in traditional Korean herbal medicine, was evaluated as a potential drug in multiple diseases and was suggested for use in the experimental therapy of $\mathrm{BPH}$ [15]. Its effect is in part based on the inhibition of adrenergic receptors in $\mathrm{BPH}$. Although the role of the environmental endocrine disruptor bisphenol needs to be investigated in more detail in appropriate $\mathrm{BPH}$ models, the evidence that it up-regulates aromatase should be taken into consideration with regard to $\mathrm{BPH}$ pathogenesis. Bisphenol is nearly ubiquitously present in the environment, including plastics and food containers. In addition to oestrogen receptor ligands, the ability of selective oestrogen receptor modulators in the inhibition of $\mathrm{BPH}$ has been described in the literature (reviewed by [16]).

In summary, therapeutic options in BPE/LUTS could be based on the antagonism between androgenic and oestrogenic hormones. Hormonal regulation also includes several peptide hormones, and the regulatory system based on IGFs seems to be the most important one. Antagonism of this system in combination with $5 \alpha$-reductase inhibitors may be a valuable option in future $\mathrm{BPH}$ treatment.

Benign Prostatic Hyperplasia

\section{Age-Related Tissue Remodelling}

Aging and androgens are the 2 established risk factors for the development of BPH/BPE [17]. In addition, local para- and luminocrine pleiotrope mechanisms/factors are implicated in the prostatic tissue remodelling process as exemplified in a review by Untergasser et al. [18]. Prostate tissue remodelling in the transition zone is characterized by (1) hypertrophic basal cells; (2) altered secretions of luminal cells leading to calcification, clogged ducts and inflammation; (3) lymphocytic infiltration with production of proinflammatory cytokines; (4) increased radical oxygen species production that damages epithelial and stromal cells; (5) increased basic fibroblast and TGF- $\beta$ production leading to stromal proliferation, transdifferentiation and extracellular matrix production; (6) altered autonomous innervation that decreases relaxation and leads to a high adrenergic tonus; and (7) altered neuroendocrine cell function and release of neuroendocrine peptides [18].

\section{Inflammation}

The observation of chronic inflammation coexisting with $\mathrm{BPH}$ histologic changes in pathologic specimens led to the suspicion that inflammation plays a role in the development of BPH/BPH and also LUTS (Fig. 2). Local inflammation may be triggered by viral or bacterial infection, 
which would lead to the secretion of cytokines, chemokines and growth factors involved in the inflammatory response with consequent growth of epithelial and stromal prostatic cells [4]. It has been hypothesized that the inflammatory response is perpetuated by the release of prostatic self-antigens following tissue damage, which would sensitize the immune system and start autoimmune responses (Fig. 2) [4]. Important factors in this process are the prostatic stromal cells, which activate $\mathrm{CD} 4^{+}$lymphocytes and proinflammatory cytokines and chemokines, such as stromalderived interleukin-8 (Fig. 2) [19].

Prostatic inflammation was associated with overall clinical progression and an increased risk of urinary retention and the need for surgery [20]. Furthermore, a positive association between high plasma $\mathrm{C}$-reactive protein levels and the odds of reporting moderate to severe LUTS was reported as well.

\section{Metabolic Factors}

Results from multiple pre-clinical and clinical studies indicate that several age-related metabolic aberrations (metabolic syndrome, obesity, dyslipidaemia and diabetes) are important determinants in both the development and the progression of BPH/LUTS (Fig. 2) [21]. Metabolic syndrome and its related comorbidities, such as sex steroid alterations and low-grade inflammation, have been related to BPH/LUTS development and progression (Fig. 2). In the Baltimore Longitudinal Study of Aging cohort, each $1 \mathrm{~kg} /$ $\mathrm{m}^{2}$ increase in body mass index (BMI) corresponds to a $0.4-\mathrm{mL}$ increase in prostate volume. Obese (BMI $>35 \mathrm{~kg} /$ $\mathrm{m}^{2}$ ) participants had a 3.5-fold increased risk of prostate enlargement compared to non-obese (BMI $<25 \mathrm{~kg} / \mathrm{m}^{2}$ ) participants [22]. Most established aspects of the metabolic syndrome are linked to $\mathrm{BPH} / \mathrm{BPE}$. The presence of metabolic syndrome is associated with a higher annual BPE growth rate, increased sympathetic activity and LUTS [4, 2]. The underlying pathophysiologic mechanisms involved in the association of metabolic factors with BPH/BPE/ LUTS are not completely understood, but systemic inflammation, pelvic ischaemia and increased sympathetic activity may play a role (Fig. 2) [4, 21].

\section{Risk Factors and Disease Prevention}

For decades there have been 2 accepted, well-established risk factors for the development of $\mathrm{BPH} / \mathrm{BPH}$ : the presence of functioning testes at the time of puberty (see above) as a required permissive element and age. The role of testosterone/DHT is further substantiated by the absence of an enlarged prostate (and also of prostate cancer) in men with a genetic defect of the $5 \alpha$-reductase type II and those castrated around the age of puberty (eunuchs).

Numerous epidemiological studies have attempted to identify correlates and risk factors for BPE/LUTS, such as religion, socioeconomic factors, sexual activity, vasectomy, cigarette smoking and alcohol. These studies failed to demonstrate a consistent association with BPE/LUTS [1, 23].

There is evidence of familial aggregation of male LUTS, although more recent studies suggest only a modest association. In the Olmsted County study, for instance, having either a father or a brother with a history of diagnosed BPE was associated with an odds ratio of 1.5 for moderate to severe LUTS at baseline, and such risks seem to be cumulative, with 2 or more affected relatives conferring a greater risk [24]. Twin studies provide estimates of heritability that are less confounded by environmental or lifestyle factors that may be shared within families. Several twin studies have confirmed a heritability of BPH/BPE/LUTS in the range of $50-70 \%$, suggesting a similar heritability as for many complex diseases [25]. A recent meta-analysis aimed to identify candidate polymorphisms/genes for $\mathrm{BPH} /$ LUTS and yielded only a few putative genetic risk variants reliably replicated across populations. This review included also the most frequently cited genetic variant in this context, the number of CAG repeats within the AR [26]. The authors observed consistent evidence of a reduced risk of LUTS associated only with the common rs731236 variant of the vitamin D receptor gene [26].

The high prevalence of the disease and its socioeconomic impact raises the issue of disease prevention. Long-term studies with the $5 a$-reductase inhibitors finasteride and dutasteride suggest that both drugs reduce the risk of acute urinary retention and the need for surgery [1]. However, for the indication "prevention" in men with no or only mild LUTS, both drugs are not licensed mainly because the risk (costs/side effects)/benefit ratio remains unclear. Whether promoting a healthier lifestyle (metabolic syndrome) can alter a man's propensity to develop BPH/LUTS remains to be clarified in large-scale, long-term controlled trials.

\section{Clinical Perspective}

The development of LUTS is a multifactorial process with $\mathrm{BPH} / \mathrm{BPE}$ and urodynamic changes being important co-factors. Another relevant aspect is the response of the 
urinary bladder to benign prostatic obstruction, i.e., the development of detrusor overactivity or detrusor hypo-/ acontractility. The most frequent urodynamic pattern in elderly men with LUTS is detrusor overactivity (60\%), while benign prostatic obstruction is only present in 30 $40 \%$ [3]. Other important mediators are inflammation, atherosclerosis, urothelial dysfunction, $\alpha$ - and anti-muscarinic receptor status, etc. [4].

The standard of care is defined by evidence-based guidelines, such as those of the European Association of Urology (EAU) [1]. Management of men with LUTS due to $\mathrm{BPH} / \mathrm{BPE}$ is driven by symptom status, objective parameters (post-void residual volume and uroflowmetry), risk of disease progression and the presence of $\mathrm{BPH}$-related complications, such as recurrent urinary retention, bladder stones or hydronephrosis/decline in renal function [1].

It is beyond the scope of this article to describe the various treatment options in more detail, and the reader is referred to, for example, the above-mentioned EAU guidelines; yet, a few principles are briefly presented [1]. All guidelines suggest a risk-adapted, individualized approach (AUA guidelines [1]). In men with mild symptoms (IPSS $<8$ ), no complications and low risk of disease progression, a conservative, watchful waiting approach is recommended. In those with moderate/severe symptoms and no complications, medical therapy is indicated [1]. The following drugs are currently in use, either alone or in various combinations: plant extracts (low level of evidence), $\alpha$-blockers (level I evidence, moderate effect on LUTS, uroflowmetry and post-void residual volume, no effect on prostate volume and disease progression), $5 \alpha$-reductase inhibitors (level I evidence, moderate effect on LUTS/uroflowmetry/post-void residual volume, 20$30 \%$ reduction of prostate volume and effect on disease progression [urinary retention/risk for surgery]), and PDE-5 inhibitors (moderate evidence, moderate effect on LUTS/uroflowmetry/post-void residual volume, no effect on prostate volume/disease progression, long-term efficacy unknown) [1]. The role of plant extracts and anti-inflammatory drugs is controversially discussed and not recommended by guidelines [1].

Within the past 2 decades, several minimally invasive procedures as alternatives to conventional surgical approaches have been developed, such as transurethral microwave thermotherapy, transurethral needle ablation, interstitial laser coagulation and, more recently, UroLift, the Rezum system, prostate embolization or aquablation [1]. Many of these techniques have been abandoned mainly because of disappointing long-term data [1].
For more than 50 years, the standard surgical procedure has been transurethral resection of the prostate with excellent long-term data unsurpassed by alternative techniques [1]. Open prostatectomy (which was the surgical standard for patients with prostates exceeding 80-100 $\mathrm{mL}$ ) is increasingly forced back by various enucleation techniques resulting in similar functional outcomes yet considerably lower morbidity, such as risk of bleeding, duration of catheterisation and hospitalisation [1]. As outlined in BPH/LUTS guidelines, the surgical approach should be individualized based on surgical risk (anaesthesiologic, bleeding), prostate volume and patient preferences (preservation of sexual function).

\section{Statement of Ethics}

The authors have no ethical conflicts to disclose.

\section{Disclosure Statement}

The authors have no conflicts of interest to declare.

\section{Funding Sources}

There are no funding sources.

\section{Author Contributions}

All authors contributed equally to this manuscript.

References

1 Gravas S, Cornu JN, Drake MJ, Gacci M, Gratzke C, Herrmann TRW, et al. Management of non-neurogenic male lower urinary tract symptoms (LUTS). Limited update March 2018. Available from: https://uroweb. org/wp-content/uploads/EAU-Guidelineson-the-Management-of-Non-neurogenicMale-LUTS-2018-large-text.pdf

2 Oelke M, Baard J, Wijkstra H, de la Rosette JJ, Jonas U, Höfner K. Age and bladder outlet obstruction are independently associated with detrusor overactivity in patients with benign prostatic hyperplasia. Eur Urol. 2008 Aug; 54(2):419-26.

3 Madersbacher S, Haidinger G, Temml C, Schmidbauer CP. Prevalence of lower urinary tract symptoms in Austria as assessed by an open survey of 2,096 men. Eur Urol. 1998 Aug;34(2):136-41. 
4 Soler R, Andersson KE, Chancellor MB, Chapple CR, de Groat WC, Drake MJ, et al. Future direction in pharmacotherapy for non-neurogenic male lower urinary tract symptoms. Eur Urol. 2013 Oct;64(4):610-21.

5 Mononen N, Ikonen T, Autio V, Rökman A, Matikainen MP, Tammela TL, et al. Androgen receptor CAG polymorphism and prostate cancer risk. Hum Genet. 2002 Aug; 111(2):166-71.

6 Carvalho-Dias E, Miranda A, Martinho O, Mota P, Costa Â, Nogueira-Silva C, et al. Serotonin regulates prostate growth through androgen receptor modulation. Sci Rep. 2017 Nov;7(1):15428.

7 Jung Y, Park J, Kim HL, Youn DH, Kang J, Lim S, et al. Vanillic acid attenuates testosterone-induced benign prostatic hyperplasia in rats and inhibits proliferation of prostatic epithelial cells. Oncotarget. 2017 Aug; 8(50): 87194-208.

8 Li F, Pascal LE, Zhou J, Zhou Y, Wang K, Parwani $\mathrm{AV}$, et al. BCL-2 and BCL-XL expression are down-regulated in benign prostate hyperplasia nodules and not affected by finasteride and/or celecoxib. Am J Clin Exp Urol. 2018 Feb;6(1):1-10.

9 Wang K, Jin S, Fan D, Wang M, Xing N, Niu Y. Anti-proliferative activities of finasteride in benign prostate epithelial cells require stromal fibroblasts and c-Jun gene. PLoS One. 2017 Feb;12(2):e0172233.

10 Alisky JM, Tang Y, Habermehl GK, Iczkowski KA. Dutasteride prevents the growth response to testosterone in benign and androgen-sensitive malignant prostate cells. Int J Clin Exp Med. 2010 Jul;3(3):245-7.
11 Mosli HH, Esmat A, Atawia RT, Shoieb SM, Mosli HA, Abdel-Naim AB. Metformin attenuates testosterone-induced prostatic hyperplasia in rats: a pharmacological perspective. Sci Rep. 2015 Oct;5(1):15639.

12 Sreenivasulu K, Nandeesha H, Dorairajan LN, Rajappa M, Vinayagam V. Elevated insulin and reduced insulin like growth factor binding protein-3/prostate specific antigen ratio with increase in prostate size in Benign Prostatic Hyperplasia. Clin Chim Acta. 2017 Jun;469:37-41.

13 Sampson N, Zenzmaier C, Heitz M, Hermann M, Plas E, Schäfer G, et al. Stromal insulin-like growth factor binding protein 3 (IGFBP3) is elevated in the diseased human prostate and promotes ex vivo fibroblast-to-myofibroblast differentiation. Endocrinology. 2013 Aug; 154(8):2586-99.

14 Ammar AE, Esmat A, Hassona MD, Tadros MG, Abdel-Naim AB, Guns ES. The effect of pomegranate fruit extract on testosterone-induced BPH in rats. Prostate. 2015 May;75(7): 679-92.

15 Kim SK, Chung JH, Lee BC, Lee SW, Lee KH, Kim YO. Influence of Panax ginseng on alphaadrenergic receptor of benign prostatic hyperplasia. Int Neurourol J. 2014 Dec;18(4):179-86.

16 Nicholson TM, Ricke WA. Androgens and estrogens in benign prostatic hyperplasia: past, present and future. Differentiation. 2011 NovDec;82(4-5):184-99.

17 Sampson N, Madersbacher S, Berger P. [Pathophysiology and therapy of benign prostatic hyperplasia]. Wien Klin Wochenschr. 2008;120(13-14):390-401.

18 Untergasser G, Madersbacher S, Berger P. Benign prostatic hyperplasia: age-related tissueremodeling. Exp Gerontol. 2005 Mar;40(3): $121-8$.
19 Chughtai B, Lee R, Te A, Kaplan S. Role of inflammation in benign prostatic hyperplasia. Rev Urol. 2011;13(3):147-50.

20 Nickel JC, Freedland SJ, Castro-Santamaria $\mathrm{R}$, Moreira DM. Chronic prostate inflammation predicts symptom progression in patients with chronic prostatitis/chronic pelvic pain. J Urol. 2017 Jul;198(1):122-8.

21 Sebastianelli A, Gacci M. Current status of the relationship between metabolic syndrome and lower urinary tract symptoms. Eur Urol Focus. 2018 Jan;4(1):25-7.

22 Parsons JK, Carter HB, Partin AW, Windham BG, Metter EJ, Ferrucci L, et al. Metabolic factors associated with benign prostatic hyperplasia. J Clin Endocrinol Metab. 2006 Jul; 91(7):2562-8.

23 de la Taille A, Descazeaud A, Robert G. [How to prevent LUTS due to BPH development and progression]. Prog Urol. 2018 Nov; 28(15):821-9.

24 Roberts RO, Rhodes T, Panser LA, Girman CJ, Chute CG, Guess HA, et al. Association between family history of benign prostatic hyperplasia and urinary symptoms: results of a population-based study. Am J Epidemiol. 1995 Nov; 142(9):965-73.

25 Partin AW, Page WF, Lee BR, Sanda MG, Miller RN, Walsh PC. Concordance rates for benign prostatic disease among twins suggest hereditary influence. Urology. 1994 Nov; 44(5):646-50.

26 Cartwright R, Mangera A, Tikkinen KA, Rajan $\mathrm{P}$, Pesonen J, Kirby AC, et al. Systematic review and meta-analysis of candidate gene association studies of lower urinary tract symptoms in men. Eur Urol. 2014 Oct;66(4):752-68. 